withdraw from the study. The primary endpoint is progression-free survival (PFS) as assessed by the investigator in the all-comers population and the dMMR population per RECIST version 1.1. Secondary efficacy endpoints are PFS assessed by blinded independent central review per RECIST version 1.1, overall survival, objective response rate, duration of response, disease control rate, safety and tolerability, and patientreported outcomes.

Disclosures Sponsor: GlaxoSmithKline, Waltham, MA, USA

NCT number: NCT03981796

Encore statement: This data is presented on behalf of the original authors with their permission. Presented at the American Society of Clinical Oncology (ASCO) Annual Meeting, May 29-31, 2020, Virtual.

Dr. Mirza reports personal fees and other from Karyopharm Therapeutics; Personal fees and other from Sera Prognostics and Roche; Grants and Personal fees from AstraZeneca, Clovis Oncology, Pfizer, GSK, Genmab, BioCad, Sotio, Boehringer Ingelheim, Geneos Therapeutics, Merck, Oncology Venture, Seattle Genetics, Sera Prognostics,Takeda Pharmaceutical Company Ltd, and Zailab.

Dr. Coleman reports consulting fees from Merck, Reoche/ Genentech, AstraZeneca, Oncomed/Mateo, Novocure, Oncosec, Janssen, Clovis, Tesaro/GSK, Abbvie, Eisai, Arrivive, and Oncoquest; grants from Merck, Roche/Genentech, V-Foundation, AstraZeneca, Janssen, Clovis, Genmab and Abbvie; and honoraria/reimbursement from Merck, Roche/Genentech, AstraZeneca, Oncomed/Mateo, Novocure, Oncosec, Janssen, Clovis, Tesaro/GSK, Eisai, Arrivive and OncoQuest.

Dr. Slomovitz reports consulting/advisory fees from GlaxoSmithKline.

Dr. Powell reports consulting/advisory fees from Roche/ Genentech, AstraZeneca, Tesaro, and Clovis Oncology; and speakers' bureau at Genentech/Roche, AstraZeneca, Tesaro and Clovis Oncology.

Drs. Hanker and Valabrega have nothing to disclose.

Drs. Im, Walker, and Guo are employees of GlaxoSmithKline.

\section{PHASE 3 TRIAL OF TUMOR TREATING FIELDS CONCOMITANT WITH WEEKLY PACLITAXEL FOR PLATINUM-RESISTANT OVARIAN CANCER: ENGOT- OV50/GOG-329/INNOVATE-3}

${ }^{1}$ Ignace B Vergote, ${ }^{2}$ Jalid Sehouli, ${ }^{3}$ Roldano Fossati, ${ }^{4}$ Robert Coleman, ${ }^{5}$ Bradley Monk, ${ }^{6}$ Larry Copeland, ${ }^{7}$ David O'malley. 'Leuven Cancer Institute, Leuven, Belgium; Leuven Cancer Institute, Leuven, Belgium; Gynaecological Oncology; ${ }^{2}$ Charité Universitätsmedizin Berlin; Klinik für Gynäkologie; ${ }^{3}$ Irccs-Istituto DI Ricerche Farmacologiche, Milan; ${ }^{4} \mathrm{MD}$ Anderson Cancer Center University of Texas; ${ }^{5}$ Arizona Oncology US Oncology Network; ${ }^{6}$ The James Cancer Hospital; Ohio State University Comprehensive Cancer Center; ${ }^{7}$ Ohio State University Comprehensive Cancer Center

\subsection{6/ijgc-2020-ESG0.198}

Introduction/Background Tumor Treating Fields (TTFields) are a non-invasive, antimitotic cancer therapy. The Phase 2 INNOVATE study demonstrated safety of TTFields/weekly paclitaxel in 31 PROC (platinum-resistant ovarian cancer) patients (Vergote Gyn Onc 2018); efficacy: median PFS 8.9 months, 25\% partial response,71\% clinical benefit and 61\% 1-year survival rate. This phase 3 ENGOT-ov50/GOG-329/INNOVATE-3 study [NCT03940196] investigates TTFields plus weekly paclitaxel in PROC patients.
Methodology Patients ( $\mathrm{N}=540)$ will have PROC (RECIST V1.1) within 6 months of last platinum therapy with maximum of 2-5 prior lines of systemic therapy, ECOG $0-1$ and no peripheral neuropathy >grade1. Patients with primary refractory disease will be excluded. Patients will be randomized 1:1 to weekly paclitaxel alone or weekly paclitaxel (starting of dose $80 \mathrm{mg} / \mathrm{m} 2$ weekly for 8 weeks, and then on Days 1,8 , and 15 for subsequent 28-day cycle ) plus TTFields (200 kHz for 18 hours/day and continued if no progression in the abdominal or pelvic regions ('in-field region') per RECIST V1.1. Clinical follow-up will be performed q4w, with radiological follow-up (CT or MRI scans of the abdomen and chest) $\mathrm{q} 8 \mathrm{w}$. The primary endpoint is overall survival. Secondary endpoints: PFS, objective response rate, AEs, and quality of life (EORTC QLQ-C30 with QLQ-OV28). Sample size $(n=540)$ will detect an increase in median OS from 12 to 16 months (HR 0.75). Data Monitoring Committee (DMC) meeting (March 2020) concluded that data to-date showed no safety issues and recommended trial continuation.

Results TiP N/A

Conclusion TiP N/A

Disclosures

\section{BIOPSAR STUDY: ULTRASOUND-GUIDED PREOPERATIVE BIOPSY TO ASSESS HISTOLOGY OF SARCOMA- SUSPICIOUS UTERINE TUMORS. A NEW STUDY PROTOCOL}

${ }^{1}$ Stamatios Petousis, ${ }^{2}$ Sabrina Croce, ${ }^{2}$ Michel Kind, ${ }^{2}$ Caroline Lalet, ${ }^{2}$ Guillame Babin, ${ }^{3}$ Chrysoula Margioula-Siarkou, ${ }^{2}$ Dennis Querleu, ${ }^{2}$ Anne Floquet, ${ }^{2}$ Marina Pulido, ${ }^{2}$ Frederic Guyon. '2nd Department of Obstetrics and Gynaecology, Aristotle University of Thessaloniki; ${ }^{2}$ Institut Bergonie, Bordeaux, France; ${ }^{3}$ Institut Bergonie, Bordeaux, France; 2nd Department of Obstetrics and Gynaecology, Aristotle University of Thessaloniki, Greece

\subsection{6/ijgc-2020-ESGO.199}

Topic: Trials in progress abstract

Introduction/Backgroun The preoperative differential diagnosis between a uterine fibroma and a sarcoma is a challenge. Available diagnostic tools are rather inconclusive to distinguish between two pathologies. However, potential accurate diagnostic methods would be of great clinical impact in order to optimize surgical treatment.

Methodology A prospective multi-center interventional study will be performed. Ten tertiary French centers will participate in the present study. The overall inclusion study period will be 2 years, overall study duration will be 5 years. Patients greater than 35 years old, diagnosed with suspicious uterine tumor and needing surgical intervention will be included. Uterine tumors will be considered as suspicious in case of rapid tumor growth $(\geq 30 \%$ of the maximum diameter in 1-year interval), symptomatic tumors in postmenopausal women, tumors characterized by certain suspicious ultrasound criteria history of treatment with tamoxifen and genetical predisposal to cancer syndromes. Included patients will undergo preoperatively a Vaginal Ultrasound-Guided Biopsy (VUGB). There will be two histopathological diagnoses for each patient, the first based on the biopsy specimen received preoperatively (Index test) and the second based on the surgical specimen of uterus resected 'en block'. These diagnoses will be compared in order to assess diagnostic performance of VUGB. Histological criteria used for both diagnoses will be that of Bell et al which were revised by OMS 2014 classification. 